\title{
Slow motion of a slip spheroid along its axis of revolution
}

\author{
H.J. Keh*, Y.C. Chang \\ Department of Chemical Engineering, National Taiwan University, Taipei 106-17, Taiwan, ROC
}

Received 11 September 2007; received in revised form 23 November 2007

\begin{abstract}
A combined analytical and numerical study of the Stokes flow caused by a rigid spheroidal particle translating along its axis of revolution in a viscous fluid is presented. The fluid is allowed to slip at the surface of the particle. The general solution for the stream function in prolate and oblate spheroidal coordinates can be expressed in an infinite-series form of semi-separation of variables. The slip boundary condition incorporating the shear stress at the particle surface is applied to this general solution to determine its unknown coefficients of the leading orders. The solution of these coefficients can be either numerical results obtained from a boundary-collocation method or explicit formulas derived analytically. The drag force exerted on the spheroidal particle by the fluid is evaluated with good convergence behavior for various values of the slip parameter and aspect ratio of the particle. The agreement between our hydrodynamic drag results and the relevant numerical solutions obtained previously using a singularity method is excellent. Although the drag force acting on the translating spheroid normalized by that on a corresponding sphere with equal equatorial radius increases monotonically with an increase in the axial-to-radial aspect ratio for a no-slip spheroid, it decreases monotonically as this aspect ratio increases for a perfect-slip spheroid. The normalized drag force exerted on a spheroid with a given surface slip coefficient in between the no-slip and perfect-slip limits is not a monotonic function of its aspect ratio. For a spheroid with a fixed aspect ratio, its drag force is a monotonically decreasing function of the slip coefficient of the particle.
\end{abstract}

(c) 2008 Elsevier Ltd. All rights reserved.

Keywords: Axisymmetric creeping flow; Prolate and oblate spheroids; Slip-flow surface; Hydrodynamic drag force

\section{Introduction}

The movement of a small particle in a fluid at low-Reynolds-numbers is of much fundamental and practical interest in the areas of chemical, biomedical, and environmental engineering and science. The theoretical treatment of this subject has grown out of the classic work of Stokes (1851) for a translating rigid sphere in an unbounded Newtonian fluid. Oberbeck (1876) extended this result to the translation of an ellipsoid. More recently, analytical solutions of the creeping-flow problem have been obtained for rigid particles whose shape corresponds to a coordinate surface of a special orthogonal curvilinear coordinate system in which the Stokes equations can be solved using the separation of variables (Payne and Pell, 1960; Goren

\footnotetext{
* Corresponding author. Tel.: +88623366 3048; fax: +886223623040. E-mail address: huan@ntu.edu.tw (H.J. Keh).
}

and O'Neill, 1980) and for long slender bodies (Batchelor, 1970; Cox, 1970). Explicit expressions for the resistance experienced by a slightly deformed sphere undergoing low-Reynolds-number motions in an unbounded fluid were also derived to the first order of the small parameter characterizing the deformation (Brenner, 1964).

In the general formulation of the Stokes problem, it is usually assumed that no slippage arises at the solid-fluid interfaces. Actually, this is an idealization of the transport processes involved. The phenomena that the adjacent fluid can slip frictionally over a solid surface, occurring in cases such as the low-density gas flow surrounding an aerosol particle (Kennard, 1938; Hutchins et al., 1995; Keh and Shiau, 2000), the aqueous liquid flow near a hydrophobic surface (Tretheway and Meinhart, 2002; Gogte et al., 2005), and the Newtonian fluid flow over a porous surface (Beavers and Joseph, 1967; Saffman, 1971; Jones, 1973; Nir, 1976), have been confirmed, both experimentally and 
theoretically. Presumably, any such slipping would be proportional to the local tangential viscous stress next to the solid surface (Happel and Brenner, 1983; Keh and Chen, 1996), known as the Navier slip (see Eq. (9b)), at least as the velocity gradient is small. The constant of proportionality, $\beta^{-1}$, is called a "slip coefficient".

Basset (1961) found that the drag force exerted by the fluid of viscosity $\eta$ on a translating rigid sphere of radius $b$ with a slip-flow boundary condition at its surface is

$F=-6 \pi \eta b U \frac{\beta b+2 \eta}{\beta b+3 \eta}$

where $U$ is the translational velocity of the particle. The quantity $\eta / \beta$ is a length, which can be pictured by noting that the fluid motion is the same as if the solid surface is displaced inward by a distance $\eta / \beta$ with the velocity gradient extending uniformly right up to no-slip velocity at the surface. In the limiting case of $\beta b / \eta \rightarrow \infty$, there is no slip at the particle surface and Eq. (1) degenerates to the well-known Stokes law. When $\beta b / \eta \rightarrow 0$, there is a perfect slip at the particle surface and the particle acts like a spherical inviscid gas bubble.

The slip coefficient in Eq. (1) has been determined experimentally for various gas-solid system and found to agree with the general kinetic theory of gases. It can be evaluated from the formula

$\beta^{-1}=\frac{C_{\mathrm{m}} l}{\eta}$,

where $l$ is the mean free path of a gas molecule, and $C_{\mathrm{m}}$ is a dimensionless constant related to the momentum accommodation coefficient at the solid surface (Kennard, 1938). Although $C_{\mathrm{m}}$ surely depends upon the nature of the surface and adjacent fluid, examinations of the experimental data and theoretical predictions suggest that it will be in the range 1.0-1.5 (Davis, 1972; Talbot et al., 1980; Sharipov and Kalempa, 2003). The reciprocal of the ratio $(\beta b+$ $2 \eta) /(\beta b+3 \eta)$ in Eq. (1) is equivalent to the so-called Cunningham correction factor for the slip effect of an aerosol sphere.

The problem of slow motion of nonspherical particles with frictionally slip surfaces is a matter of great analytical difficulty and was usually estimated by an adjusted sphere approximation (Dahneke, 1973). This simple approximation comprises calculating the radius of the adjusted sphere which has the same slip correction factor as the nonspherical particle. On the other hand, the axisymmetric creeping flow of a viscous incompressible fluid past a slip spheroid which departs slightly in shape from a sphere was investigated by several researchers (Palaniappan, 1994; Ramkissoon, 1997), and an explicit expression for the drag force experienced by the particle was given to the first order in the small parameter characterizing the deformation of the particle from the spherical shape. However, their approach was wrong from the very onset, since they chose an incorrect boundary condition on the surface of the slightly deformed sphere, as pointed out by Senchenko and Keh
(2006), who analyzed the translation and rotation of a slightly deformed slip sphere in arbitrary directions. The slow motion caused by a general axisymmetric particle with a slip surface translating along its axis of revolution was also numerically examined to some extent by using a method of internal singularity distributions (Keh and Huang, 2004). However, the problem of the slow motion of a general slip spheroid in a viscous fluid has not been analytically solved yet, mainly due to the fact that, if momentum slip is included, a simple separation-of-variable solution is not feasible for the prolate and oblate spheroidal coordinate systems (Leong, 1984; Williams, 1986).

In the present work, a semi-separable general solution in the form of an infinite series expansion for the axisymmetric creeping flow in spheroidal coordinates developed by Dassios et al. (1994) is used to investigate the Stokes problem of a slip prolate or oblate spheroidal particle translating steadily along its axis of revolution. The drag force exerted on the spheroid by the ambient fluid as a function of the slip parameter and aspect ratio of the spheroid can be expressed in an approximate but explicit form and calculated numerically using a boundary-collocation method. Our drag results show excellent agreement with the available analytical and numerical solutions obtained previously.

\section{Analysis}

We consider the translational motion of a spheroidal particle along its axis of revolution in an incompressible, Newtonian fluid at the steady state, as shown in Fig. 1.

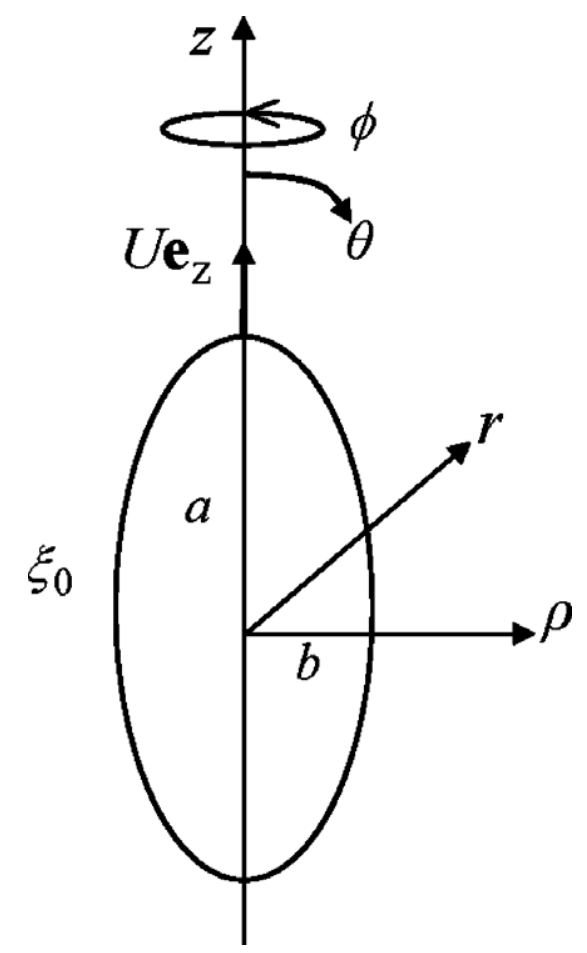

Fig. 1. Geometrical sketch for the motion of a spheroidal particle along its axis of revolution. 
The fluid may slip frictionally at the surface of the particle and is at rest at infinity. For convenience, circular cylindrical coordinates $(\rho, \phi, z)$ are established such that the surface of the spheroid is represented by

$\frac{z^{2}}{a^{2}}+\frac{\rho^{2}}{b^{2}}=1$,

where $a$ and $b$ are the half-length along the axis of revolution and the equatorial radius, respectively, of the spheroid. The particle velocity equals $U \mathbf{e}_{z}$, where $\mathbf{e}_{z}$ is the unit vector in the positive $z$-direction.

The Reynolds number is assumed to be sufficiently small so that the inertial terms in the fluid momentum equation can be neglected in comparison with the viscous terms. Therefore, the fluid motion is governed by the steady fourth-order differential equation for viscous axisymmetric creeping flows,

$E^{4} \Psi=E^{2}\left(E^{2} \Psi\right)=0$,

in which the Stokes stream function $\Psi(\lambda, \omega)$ is related to the velocity components (with $v_{\phi}=0$ ) in right-handed bifocal coordinates $(\xi, \varphi, \phi)$ by (Happel and Brenner, 1983)

$v_{\lambda}=\frac{1}{c^{2} \sqrt{\lambda^{2}-\omega^{2}} \sqrt{\lambda^{2}-1}} \frac{\partial \Psi}{\partial \omega}$

$v_{\omega}=\frac{1}{c^{2} \sqrt{\lambda^{2}-\omega^{2}} \sqrt{1-\omega^{2}}} \frac{\partial \Psi}{\partial \lambda}$,

and the Stokes operator $E^{2}$ has the form

$E^{2}=\frac{1}{c^{2}\left(\lambda^{2}-\omega^{2}\right)}\left[\left(\lambda^{2}-1\right) \frac{\partial^{2}}{\partial \lambda^{2}}+\left(1-\omega^{2}\right) \frac{\partial^{2}}{\partial \omega^{2}}\right]$.

Here, $\omega$ and $\lambda$ are the variables and $c$ is a constant related to the bifocal-coordinate transformations.

For prolate spheroids $(b<a)$, the coordinate transformation used is

$$
\begin{aligned}
& \lambda=\cosh \xi, \quad \omega=\cos \varphi, \\
& z=c \cosh \xi \cos \varphi, \quad \rho=c \sinh \xi \sin \varphi, \\
& a=c \cosh \xi_{0}, \quad b=c \sinh \xi_{0}, \\
& c=\left(a^{2}-b^{2}\right)^{1 / 2}
\end{aligned}
$$

while for oblate spheroids $(b>a)$, the coordinate transformation becomes

$$
\begin{aligned}
& \lambda=i \sinh \xi, \quad \omega=\cos \varphi, \\
& z=\mathrm{i} c \sinh \xi \cos \varphi, \quad \rho=\mathrm{i} c \cosh \xi \sin \varphi, \\
& a=\mathrm{i} c \sinh \xi_{0}, \quad b=\mathrm{i} c \cosh \xi_{0}, \\
& c=-\mathrm{i}\left(b^{2}-a^{2}\right)^{1 / 2} .
\end{aligned}
$$

The origin (midpoint between the foci) of the bifocal coordinates (with $0 \leqslant \xi<\infty$ and $0 \leqslant \varphi \leqslant \pi$ ) is also set at the center of the spheroid, and the coordinate surface $\lambda=\lambda_{0}=\left[1-(b / a)^{2}\right]^{-1 / 2}$ (or $\left.\xi=\xi_{0}\right)$ corresponds to the surface of the spheroid defined by Eq. (3).

There exists a frictional slip velocity along the particle surface and the fluid flow vanishes far from the particle.
Hence, the boundary conditions for the flow field are (Happel and Brenner, 1983)

$$
\begin{aligned}
& \left.\begin{array}{l}
v_{\lambda}=0 \\
v_{\omega}=\frac{1}{\beta} \tau_{\lambda \omega}
\end{array}\right\} \quad \text { on } \quad \lambda=\lambda_{0}, \\
& \Psi \rightarrow \frac{1}{2} U c^{2}\left(\lambda^{2}-1\right)\left(1-\omega^{2}\right) \quad \text { as } \quad \lambda \rightarrow \infty,
\end{aligned}
$$

where $1 / \beta$ is the frictional slip coefficient, $\tau_{\lambda \omega}$ is the fluid shear stress expressed as

$\tau_{\lambda \omega}=\eta\left[\sqrt{\lambda^{2}-1} \frac{\partial}{\partial \lambda}\left(h v_{\omega}\right)-\sqrt{1-\omega^{2}} \frac{\partial}{\partial \omega}\left(h v_{\lambda}\right)\right]$,

and $h=c^{-1}\left(\lambda^{2}-\omega^{2}\right)^{-1 / 2}$ is a metric coefficient in bifocal coordinates. Note that Eq. (9) and (10) take a reference frame that the particle is at rest and the velocity of the fluid at infinity is the particle velocity in the opposite direction.

A general solution of the Stokes Eq. (4) in bifocal coordinates has been obtained by Dassios et al. (1994) in a series expansion of semi-separable form as

$\Psi(\lambda, \omega)=U c^{2} \sum_{k=1}^{\infty} g_{2 k}(\lambda) G_{2 k}(\omega)$,

where

$$
\begin{aligned}
g_{2}(\lambda)= & A_{1} G_{1}(\lambda)+C_{2} G_{2}(\lambda)+D_{2} H_{2}(\lambda)+A_{4} G_{4}(\lambda) \\
& +B_{4} H_{4}(\lambda) \\
g_{n}(\lambda)= & A_{n} G_{n-2}(\lambda)+B_{n} H_{n-2}(\lambda)+C_{n} G_{n}(\lambda) \\
& +D_{n} H_{n}(\lambda)+A_{n+2} G_{n+2}(\lambda) \\
& +B_{n+2} H_{n+2}(\lambda) \text { for } n=4,6,8, \ldots
\end{aligned}
$$

In the above equations, $G_{n}(\lambda)$ and $H_{n}(\lambda)$ denote the Gegenbauer functions of the first and second kinds, respectively, of order $n$ and degree $-1 / 2$, and $A_{n}, B_{n}, C_{n}$, and $D_{n}$ are unknown coefficients to be determined. Note that each individual term in Eq. (12) is not a solution of Eq. (4), whereas the entire expansion, which is not a complete separation of the variables $\omega$ and $\lambda$, is. Because the stream function is symmetric about the equatorial plane $z=0$, only the even terms of the expansion in Eq. (12) are retained. In accordance with Eq. (10), we immediately find that $C_{2}=-2$ and $A_{n}=C_{n}=0$ for $n \geqslant 4$ in Eqs. (13). Note that, in the previous studies of some relevant problems (Dassios et al., 1994, 1995; Deo and Datta, 2002), the coefficient $B_{4}$ was taken to be zero and their results are applicable only for the case of axisymmetric motion of a slightly deformed sphere (with $a / b \rightarrow 1$ ). In fact, the article by Deo and Datta (2002) contains some major errors in the determination of the nonzero unknown coefficients in Eqs. (13).

Applying Eqs. (5a) and (12) to boundary condition (9a), we obtain

$\sum_{k=1}^{\infty} g_{2 k}\left(\lambda_{0}\right) P_{2 k-1}(\omega)=0$, 
where $P_{n}(\omega)$ is the Legendre polynomial of order $n$. From the orthogonal characteristic of the Legendre polynomials, Eq. (14) is equivalent to

$g_{n}\left(\lambda_{0}\right)=0$ for $n=2,4,6, \ldots$,

and the dependence on $\omega$ disappears. The substitution of Eqs. (5), (11), (12), (13), and (15) into the slip boundary condition $(9 b)$ at the particle surface leads to

$\sum_{k=1}^{\infty} h_{2 k}\left(\lambda_{0}, \omega\right) G_{2 k}(\omega)=0$

where

$$
\begin{aligned}
h_{n}(\lambda, \omega)= & \frac{\eta}{\beta c}\left[\left(\lambda^{2}-\omega^{2}\right) g_{n}^{\prime \prime}(\lambda)-2 \lambda g_{n}^{\prime}(\lambda)\right] \\
& -\frac{\left(\lambda^{2}-\omega^{2}\right)^{3 / 2}}{\sqrt{\lambda^{2}-1}} g_{n}^{\prime}(\lambda) \text { for } n=2,4,6, \ldots,
\end{aligned}
$$

and a prime on $g_{n}(\lambda)$ denotes a differentiation with respect to $\lambda$. The unknown coefficients $A_{1}, D_{2}, B_{4}, D_{4}, B_{6}$, etc., in Eqs. (13) are to be determined using Eqs. (15) and (16).

The drag force $F \mathbf{e}_{z}$ exerted by the fluid on the particle can be determined from (Payne and Pell, 1960; Happel and Brenner, 1983)

$F=8 \pi \eta c \lim _{\lambda \rightarrow \infty} \lambda\left(\frac{\Psi}{\rho^{2}}-\frac{U}{2}\right)$.

The substitution of Eqs. (12) and (13) into Eq. (18) yields

$F=-4 \pi \eta c U A_{1}$.

This expression shows that only the lowest-order coefficient $A_{1}$ contributes to the hydrodynamic force acting on the particle.

In the following two subsections, we present a boundary-collocation method to obtain a numerical solution for the unknown coefficients $A_{1}, D_{2}, B_{4}, D_{4}, B_{6}$, etc., in Eqs. (13) and an analytical method to result in explicit formulas for these coefficients of leading orders.

\subsection{Boundary-collocation method}

To satisfy the boundary conditions (15) and (16) exactly along the entire semi-elliptic generating arc of the spheroid in a meridian plane would require the solution of the entire infinite array of the unknown constants $A_{1}, D_{2}, B_{4}, D_{4}$, $B_{6}$, etc. However, the boundary-collocation technique (O'Brien, 1968; Gluckman et al., 1971) enforces the boundary conditions at a finite number of discrete points on the particle's quarter-elliptic longitudinal arc (from $\varphi=0$ to $\pi / 2$, owing to the symmetry of the system geometry) and truncates the infinite series in Eqs. (12) and (16) into finite ones. The unknown constants in the terms of the finite series permit one to satisfy the exact boundary conditions at the discrete points on the particle surface. Thus, if the boundary is approximated by satisfying condition (16) at $N$ discrete points, then the infinite series are truncated after $N$ terms, resulting in a system of $2 N$ simultaneous linear algebraic equations [including first $N$ equations of condition (15)]. This matrix equation can be solved by any of the standard matrix-reduction techniques to yield the $2 N$ unknown constants $A_{1}, D_{2}, B_{4}, D_{4}, B_{6}$, etc., required in the truncated Eq. (12) for the flow field.

Actually, the system resulting from the boundary-collocation has $2 N+1$ unknown constants but only $2 N$ algebraic equations. Even so, this problem can be solved if the last unknown constant $B_{2 N+2}$ is negligible. Of course, the more terms are retained in Eq. (12), the less error will be caused by omitting the coefficient $B_{2 N+2}$. The accuracy of the truncation technique can also be improved to any degree by taking a sufficiently large value of $N$. Naturally, the truncation (including omission of the coefficient $B_{2 N+2}$ ) error vanishes as $N \rightarrow \infty$.

\subsection{Analytical method}

On the other hand, an analytical solution for the leading unknowns $A_{1}, D_{2}, B_{4}, D_{4}, B_{6}$, etc., required in Eq. (12) for the flow field can be found. To simplify the boundary condition given by Eqs. (16) and (17), we first expand the function $\left(\lambda_{0}^{2}-\omega^{2}\right)^{3 / 2}$ into a Taylor series in $\exp \left(-\xi_{0}\right)$ with respect to $\xi_{0} \rightarrow \infty$ (only odd terms are nonzero) and then apply the recurrence relation (Dassios et al., 1994)

$$
\begin{aligned}
& \omega^{2} G_{n}(\omega)=\alpha_{n} G_{n-2}(\omega)+\gamma_{n} G_{n}(\omega)+\beta_{n} G_{n+2}(\omega) \\
& \quad \text { for } n \geqslant 2,
\end{aligned}
$$

with

$$
\begin{aligned}
& \alpha_{n}=\frac{(n-3)(n-2)}{(2 n-3)(2 n-1)}, \\
& \beta_{n}=\frac{(n+1)(n+2)}{(2 n-1)(2 n+1)}, \\
& \gamma_{n}=\frac{\left(2 n^{2}-2 n-3\right)}{(2 n-3)(2 n+1)},
\end{aligned}
$$

to result in the expansion

$$
\frac{\left(\lambda_{0}^{2}-\omega^{2}\right)^{3 / 2}}{\sqrt{\lambda_{0}^{2}-1}} G_{m}(\omega)=\sum_{k=1}^{\infty} s_{m(2 k)} G_{2 k}(\omega),
$$

where the coefficients $s_{m n}$ are known functions of $\exp \left(-\xi_{0}\right)$ with the first ones given by Eq. (A.6) in Appendix A. Substituting Eq. (22) into Eqs. (16) and (17), applying Eq. (20) again, and using the orthogonal property of the Gegenbauer polynomials, we obtain

$$
\begin{aligned}
& \frac{\eta}{\beta c}\left[\left(\lambda_{0}^{2}-\gamma_{n}\right) g_{n}^{\prime \prime}\left(\lambda_{0}\right)-2 \lambda_{0} g_{n}^{\prime}\left(\lambda_{0}\right)-\beta_{n-2} g_{n-2}^{\prime \prime}\left(\lambda_{0}\right)\right. \\
& \left.\quad-\alpha_{n+2} g_{n+2}^{\prime \prime}\left(\lambda_{0}\right)\right]=\sum_{k=1}^{\infty} s_{(2 k)} g_{2 k}^{\prime}\left(\lambda_{0}\right) \quad \text { for } n=2,4,6, \ldots
\end{aligned}
$$

in which the dependence on $\omega$ disappears.

If Eq. (12) for the fluid flow is truncated after $N$ terms, the first $N$ equations of each of Eqs. (15) and (23) can be 
used to solve the $2 N$ unknown constants $A_{1}, D_{2}, B_{4}, D_{4}, B_{6}$, etc. Similar to the case in the previous subsection, the constant $B_{2 N+2}$ is neglected in the solution and the accuracy will be acceptable when the value of $N$ is sufficiently large. Again, the truncation error disappears as $N \rightarrow \infty$. In Appendix $\mathrm{A}$, the four algebraic equations required to solve the unknown coefficients $A_{1}, D_{2}, B_{4}$, and $D_{4}$ and their explicit solution are given for a specific case that Eq. (12) is truncated after two terms $(N=2)$. Although we have also obtained the explicit solution for the unknown coefficients $A_{1}, D_{2}, B_{4}, D_{4}, B_{6}$, and $D_{6}$ for the more accurate case of $N=3$, its formulas are not presented here for conciseness.

\section{Results and discussion}

In this section, we first present the numerical results of the hydrodynamic drag force acting on a slip spheroidal particle undergoing steady translation along its axis of revolution obtained by using the semi-separable general solution combined with the boundary-collocation method described in Section 2.1. Then, the leading-order asymptotic solutions for this drag force resulting from the analytical method introduced in Section 2.2 will be given and compared with the convergent collocation solutions.

\subsection{Boundary-collocation solutions}

The system of linear algebraic equations to be solved for the coefficients $A_{1}, D_{2}, B_{4}, D_{4}, B_{6}$, etc., using the boundarycollocation method is constructed from Eqs. (15) and (16). When specifying the $N$ points along the quarter-elliptic generating arc of the spheroid where the boundary condition $(9 a, b)$ or $(16)$ is to be exactly satisfied, the first point that should be chosen is $\varphi=\pi / 2$ ( or $\omega=0$ ), since this point defines the projected area of the particle normal to the direction of motion. In addition, the point $\varphi=0$ (or $\omega=1$ ) is also important. However, an examination of the system of linear algebraic equations in the truncated form of Eq. (16) shows that the matrix equation becomes singular if these points are used. To overcome this difficulty, these points are replaced by closely adjacent points, i.e., $\varphi=\delta$ and $\pi / 2-\delta$ (Gluckman et al., 1971). Additional points along the boundary are selected to divide the quarter-elliptic arc of the spheroid into segments with equal angles in $\varphi$. The optimum value of $\delta$ in this work is found to be $0.01^{\circ}$, with which the numerical results of the hydrodynamic drag force acting on the particle converge satisfactorily.

In Tables 1 and 2, the boundary-collocation results of $-F / 6 \pi \eta b U$, the hydrodynamic drag force for the axisymmetric motion of a prolate spheroid and an oblate spheroid, respectively, normalized by that of a sphere with equal equatorial radius are presented for several values of the axial-to-radial aspect ratio $a / b$ and the slip parameter $\beta b / \eta$. All of the results were obtained by increasing the number of collocation points $N$ until the convergence of four significant digits is achieved. The exact analytical solu-
Table 1

Boundary-collocation results of the dimensionless drag force for the motion of a prolate spheroid along its axis of revolution for various values of the aspect ratio and slip parameter of the spheroid

\begin{tabular}{llllll}
\hline$\beta b / \eta$ & $N$ & \multicolumn{2}{c}{$-F / 6 \pi \eta b U$} & & \\
\cline { 3 - 5 } & & $a / b=1.1$ & $a / b=2$ & $a / b=5$ & $a / b=10$ \\
\hline$\infty$ & 2 & 1.0201 & 1.2039 & 1.7848 & 2.6471 \\
& 3 & 1.0201 & 1.2039 & 1.7848 & 2.6471 \\
& 4 & 1.0201 & 1.2039 & 1.7848 & 2.6471 \\
& Exact solution & 1.0201 & 1.2039 & 1.7848 & 2.6471 \\
10 & 13 & 0.9415 & 1.1163 & 1.6783 & 2.5144 \\
& 14 & 0.9415 & 1.1163 & 1.6783 & 2.5145 \\
& 15 & 0.9415 & 1.1163 & 1.6783 & 2.5145 \\
& Singularity solution & 0.9415 & 1.1163 & 1.6783 & \\
1 & 14 & 0.7520 & 0.8141 & 1.1750 & 1.8047 \\
& 15 & 0.7520 & 0.8141 & 1.1750 & 1.8048 \\
& 16 & 0.7520 & 0.8141 & 1.1750 & 1.8048 \\
& Singularity solution & 0.7520 & 0.8141 & 1.1750 & \\
0 & 19 & 0.6537 & 0.5635 & 0.4027 & 0.2834 \\
& 20 & 0.6537 & 0.5635 & 0.4027 & 0.2835 \\
& 21 & 0.6537 & 0.5635 & 0.4027 & 0.2835 \\
& Singularity solution & 0.6537 & 0.5635 & 0.3999 & \\
\hline
\end{tabular}

Exact solutions and singularity solutions are obtained from Happel and Brenner (1983) and Keh and Huang (2004), respectively.

tions for the axisymmetric motion of a no-slip spheroid (with $\beta b / \eta \rightarrow \infty$ ) obtained from Happel and Brenner (1983) are also given in these tables for comparison. It can be seen that our results from the boundary-collocation method agree excellently with the exact solutions in this limit. In general, the convergence behavior of the method is quite good, even for the relatively difficult case of quite large or quite small aspect ratio $a / b$ and very small slip parameter $\beta b / \eta$.

Recently, Keh and Huang (2004) investigated the problem of slow translation of an axisymmetric slip particle along its axis of revolution using a method of internal singularity distribution combined with the boundary-collocation technique. Their values of the dimensionless drag force $-F / 6 \pi \eta b U$ for a slip spheroid (only available for the aspect ratio in the range $0.2 \leqslant a / b \leqslant 5$ ) are also listed in Tables 1 and 2 for comparison. As one can see in these tables, our results agree very well with those derived from the singularity method, except for the case that $a / b=5$ and $\beta b / \eta=0$, in which the value given by Keh and Huang is not a convergent result. Note that, when the singularity method is used, the numerical solutions only converge in a narrow range of the number of the retained terms in the infinite-series general solution and the number of the divided segments for the distributed singularities. However, this convergence problem is entirely eliminated if we use the current method with Eq. (12) for the general solution in an expansion form of semi-separation of variables.

Our numerical results of the dimensionless drag force $-F / 6 \pi \eta b U$ for the axisymmetric motion of a prolate spheroid and an oblate spheroid as a function of the aspect ratio $a / b$ for several different values of the slip parameter $\beta b / \eta$ 
Table 2

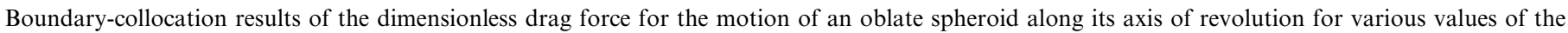
aspect ratio and slip parameter of the spheroid

\begin{tabular}{|c|c|c|c|c|c|}
\hline \multirow[t]{2}{*}{$\beta b / \eta$} & \multirow[t]{2}{*}{$N$} & \multicolumn{4}{|l|}{$-F / 6 \pi \eta b U$} \\
\hline & & $a / b=0.9$ & $a / b=0.5$ & $a / b=0.2$ & $a / b=0.1$ \\
\hline \multirow[t]{4}{*}{$\infty$} & 2 & 0.9801 & 0.9053 & 0.8615 & 0.8525 \\
\hline & 3 & 0.9801 & 0.9053 & 0.8615 & 0.8525 \\
\hline & 4 & 0.9801 & 0.9053 & 0.8615 & 0.8525 \\
\hline & Exact solution & 0.9801 & 0.9053 & 0.8615 & 0.8525 \\
\hline \multirow[t]{4}{*}{10} & 13 & 0.9052 & 0.8448 & 0.8316 & 0.8397 \\
\hline & 14 & 0.9052 & 0.8448 & 0.8316 & 0.8396 \\
\hline & 15 & 0.9052 & 0.8448 & 0.8316 & 0.8396 \\
\hline & Singularity solution & 0.9052 & 0.8448 & 0.8316 & \\
\hline \multirow[t]{4}{*}{1} & 16 & 0.7496 & 0.7696 & 0.8157 & 0.8356 \\
\hline & 17 & 0.7496 & 0.7696 & 0.8157 & 0.8355 \\
\hline & 18 & 0.7496 & 0.7696 & 0.8157 & 0.8355 \\
\hline & Singularity solution & 0.7496 & 0.7696 & 0.8157 & \\
\hline \multirow[t]{4}{*}{0} & 15 & 0.6804 & 0.7470 & 0.8122 & 0.8348 \\
\hline & 16 & 0.6804 & 0.7470 & 0.8122 & 0.8347 \\
\hline & 17 & 0.6804 & 0.7470 & 0.8122 & 0.8347 \\
\hline & Singularity solution & 0.6804 & 0.7470 & 0.8123 & \\
\hline
\end{tabular}

Exact solutions and singularity solutions are obtained from Happel and Brenner (1983) and Keh and Huang (2004), respectively.

are plotted in Figs. 2 and 3, respectively. As indicated in Eq. (1), this dimensionless force equals $(\beta b / \eta+2) /(\beta b /$ $\eta+3$ ) for the special case of a slip sphere (with $a / b=1$ ).

For a prolate spheroid with a no-slip surface $(\beta b /$ $\eta \rightarrow \infty)$ or a slip surface having large values of $\beta b / \eta$ (greater than about 1), as shown in Fig. 2, the value of $-F / 6 \pi \eta b U$ increases monotonically with an increase in the value of $a / b$. For a slip prolate spheroid with a small but finite value of $\beta b / \eta$ (less than about 1), however, $-F / 6 \pi \eta b U$ first decreases with an increase in $a / b$, reaches a minimum, and then increases monotonically with a further

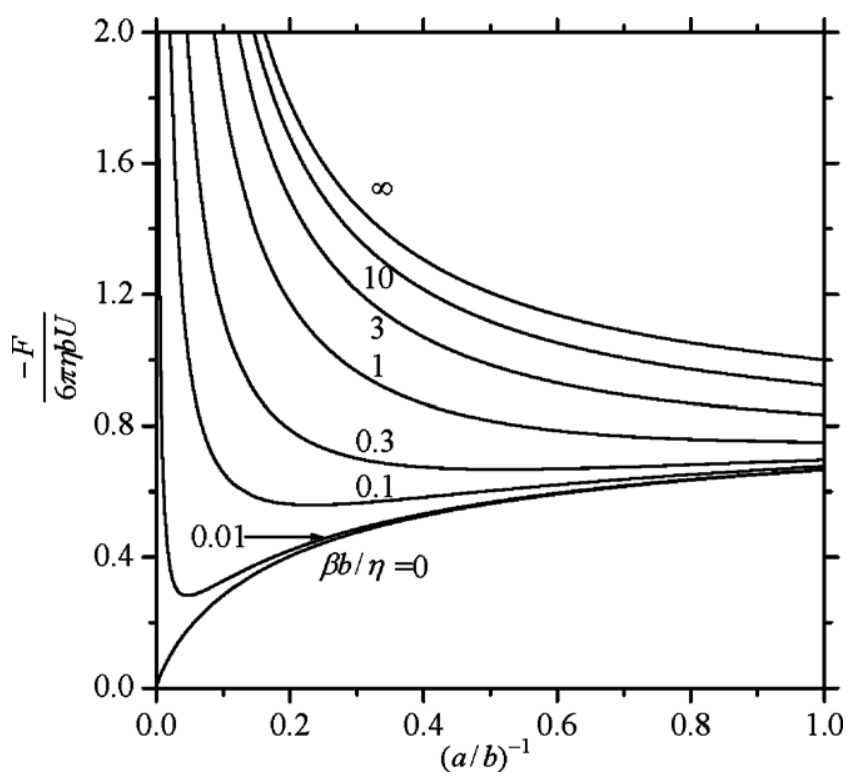

Fig. 2. Plots of the dimensionless drag force for the motion of a prolate spheroid with a slip surface along its axis of revolution versus the aspect ratio of the spheroid for various values of the slip parameter $\beta b / \eta$. increase in $a / b$. This behavior is understandable since the major portion of the fluid slip at the particle surface occurs in the direction of the particle's movement, which causes a smaller viscous retardation (in comparison with the case of a slip sphere of radius $b$ ), as the aspect ratio $a / b$ increases from unity. When $a / b$ becomes larger for a specified value of the equatorial radius $b$, however, the effect of the increase in the surface area (which experiences the hydrodynamic drag) of the prolate spheroid in comparison with a sphere of radius $b$ dominates, and the dimensionless drag increases with an increase in $a / b$. For the particular

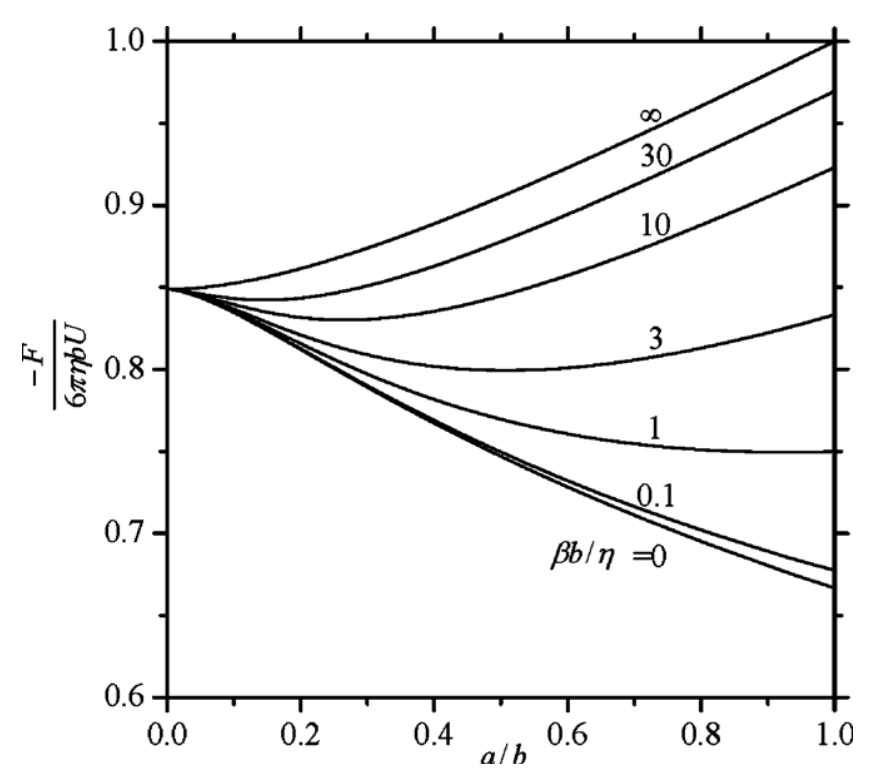

Fig. 3. Plots of the dimensionless drag force for the motion of an oblate spheroid with a slip surface along its axis of revolution versus the aspect ratio of the spheroid for various values of the slip parameter $\beta b / \eta$. 
case of a perfect-slip spheroid (with $\beta b / \eta=0$ ), the effect of the fluid slip at the particle surface is always more significant than that of the increase in the surface area, thus the dimensionless drag force decreases monotonically with an increase in $a / b$ and vanishes in the limit $a / b \rightarrow \infty$. As expected, $-F / 6 \pi \eta b U$ decreases monotonically with a decrease in the slip parameter $\beta b / \eta$ (or an increase in the surface slip coefficient $\beta^{-1}$ ) for a given shape of prolate spheroids, and this dependence becomes quite sensitive when the value of $a / b$ is large (say, greater than 2.5).

For an oblate spheroid with a no-slip surface $(\beta b /$ $\eta \rightarrow \infty)$, as illustrated in Fig. 3, the value of the dimensionless drag force $-F / 6 \pi \eta b U$ decreases monotonically as the ratio $a / b$ decreases, because of the reduction in the surface area for a given value of $b$. For an oblate spheroid with a slip surface having large but finite values of $\beta b / \eta$ (greater than about 1), the dimensionless drag first decreases with a decrease in $a / b$, reaches a minimum, and then increases with a further decrease in $a / b$. For a slip oblate spheroid with small values of $\beta b / \eta$ (less than about 1 ), the value of $-F / 6 \pi \eta b U$ increases monotonically with a decrease in $a /$ $b$. This behavior is understood since the main component of the fluid slip at the particle surface is in the direction normal to the motion of the spheroid, which has little contribution to reduce the viscous retardation (in comparison with the case of a slip sphere) when the value of $a / b$ becomes small. Similar to the case of prolate spheroids, $-F / 6 \pi \eta b U$ is a monotonically increasing function of $\beta b / \eta$ for oblate spheroids with a fixed value of $a / b$, and its dependence becomes less sensitive when the value of $a / b$ is smaller. In the limit $a / b=0,-F / 6 \pi \eta b U$ equals a constant $8 / 3 \pi \cong 0.849$, regardless of the value of $\beta b / \eta$.

\subsection{Asymptotic analytical solutions}

As discussed in Section 2.2, the coefficients $A_{1}, D_{2}, B_{4}$, $D_{4}, B_{6}$, etc., in Eqs. (12) and (13) for the Stokes flow induced by a spheroid translating along its axis of revolution can be solved explicitly using Eqs. (15) and (23) when the infinite series are truncated into finite $N$ terms. Although the truncation error disappears as $N \rightarrow \infty$, only the approximate solutions with small values of $N$ can be obtained in practice. In Tables 3 and 4, we present the asymptotic results with $N=2$ and $N=3$ (although some cases of $N>3$ can also be obtained, only the case of $N=2$ is formulated in Appendix A for conciseness) of the nondimensional drag force $-F / 6 \pi \eta b U$ for the axisymmetric motion of a prolate spheroid and an oblate spheroid for various values of the aspect ratio $a / b$ and the slip parameter $\beta b / \eta$. The relevant boundary-collocation solutions obtained in the previous subsection is also listed in these tables for comparison. One can see that the larger the value of $N$ is (say, $N=3$, where the analytical solution agrees well with the numerical solution for cases of a moderate aspect ratio in the range $0.5 \leqslant a / b \leqslant 2$ ), the more accurate the results will be. When the aspect ratio $a / b$ deviates much from unity, the agreement between the approx-
Table 3

Leading-order asymptotic results of the dimensionless drag force for the motion of a prolate spheroid along its axis of revolution for various values of the aspect ratio and slip parameter of the spheroid

\begin{tabular}{|c|c|c|c|c|c|}
\hline \multirow[t]{2}{*}{$\beta b / \eta$} & \multirow[t]{2}{*}{$N$} & \multicolumn{4}{|c|}{$-F / 6 \pi \eta b U$} \\
\hline & & $a / b=1.1$ & $a / b=2$ & $a / b=3$ & $a / b=5$ \\
\hline \multirow[t]{3}{*}{10} & 2 & 0.9415 & 1.1165 & 1.3110 & 1.6821 \\
\hline & 3 & 0.9415 & 1.1163 & 1.3101 & 1.6793 \\
\hline & Collocation solution & 0.9415 & 1.1163 & 1.3100 & 1.6783 \\
\hline \multirow[t]{3}{*}{1} & 2 & 0.7520 & 0.8126 & 0.9192 & 1.1677 \\
\hline & 3 & 0.7520 & 0.8139 & 0.9227 & 1.1697 \\
\hline & Collocation solution & 0.7520 & 0.8141 & 0.9242 & 1.1750 \\
\hline \multirow[t]{3}{*}{0} & 2 & 0.6537 & 0.5603 & 0.4790 & 0.3605 \\
\hline & 3 & 0.6537 & 0.5631 & 0.4908 & 0.3853 \\
\hline & Collocation solution & 0.6537 & 0.5635 & 0.4944 & 0.4027 \\
\hline
\end{tabular}

Table 4

Leading-order asymptotic results of the dimensionless drag force for the motion of an oblate spheroid along its axis of revolution for various values of the aspect ratio and slip parameter of the spheroid

\begin{tabular}{llllll}
\hline$\beta b / \eta$ & $N$ & \multicolumn{4}{l}{$-F / 6 \pi \eta b U$} \\
\cline { 3 - 6 } & & $a / b=0.9$ & $a / b=0.7$ & $a / b=0.5$ & $a / b=0.2$ \\
\hline 10 & 2 & 0.9052 & 0.8719 & 0.8445 & 0.8240 \\
& 3 & 0.9052 & 0.8719 & 0.8447 & 0.8272 \\
& Collocation solution & 0.9052 & 0.8719 & 0.8448 & 0.8316 \\
1 & 2 & 0.7496 & 0.7544 & 0.7676 & 0.7941 \\
& 3 & 0.7496 & 0.7546 & 0.7694 & 0.8078 \\
& Collocation solution & 0.7496 & 0.7546 & 0.7696 & 0.8157 \\
0 & 2 & 0.6804 & 0.7109 & 0.7446 & 0.7885 \\
& 3 & 0.6805 & 0.7111 & 0.7467 & 0.8039 \\
& Collocation solution & 0.6804 & 0.7111 & 0.7470 & 0.8122 \\
\hline
\end{tabular}

imate values of the hydrodynamic drag force with $N=3$ and the convergent collocation results is not as good as the cases with $a / b$ close to unity. For the limiting case of no-slip spheroids (with $\beta b / \eta \rightarrow \infty$ ), only the first term of the infinite series in Eq. (12) is nonzero, and the explicit expression for the drag force derived from Eqs. (15) and (23) is exactly the same as that given in Happel and Brenner (1983) using the separable form general solution in spheroidal coordinates.

\section{Concluding remarks}

In this paper, the boundary-collocation numerical solutions and asymptotic analytical solutions for the drag force experienced by a rigid spheroidal particle translating slowly and steadily along its axis of revolution in a viscous fluid are obtained, where the fluid may slip at the particle surface. The general solution for the Stokes stream function in spheroidal coordinates is expressed as an infinite series expansion in a semi-separable form, in which the unknown coefficients can be determined analytically to their leading orders and numerically with excellent convergence. It has 
been found that, for various values of the slip parameter $\beta b / \eta$ and axial-to-radial aspect ratio $a / b$ of the prolate or oblate spheroidal particle, the agreement between our results of the hydrodynamic drag force and the available numerical solutions obtained by using a singularity method is quite good. Although the normalized drag force $-F /$ $6 \pi \eta b U$ acting on the translating spheroid increases monotonically with an increase in the aspect ratio $a / b$ for a noslip spheroid, it decreases monotonically as this aspect ratio increases for a perfect-slip spheroid. The hydrodynamic drag force exerted on a spheroid with intermediate values of its slip parameter is not a monotonic function of its aspect ratio $a / b$. For a spheroid with a given aspect ratio, its drag force is a monotonically increasing function of the slip parameter $\beta b / \eta$ of the spheroid.

It is worth repeating that an exact analytical solution for a problem of slow motion of a spheroid with a frictional slip surface in a viscous fluid is not feasible since a complete separation-of-variable general solution for the Stokes flow does not exist in prolate or oblate spheroidal coordinates. This difficulty has been resolved to a great extent in the present study through the use of a method of semi-separation of variables (it turns out that the first term of the series solution for the stream function is sufficient for the no-slip case). An analytical solution for the motion of a spheroid along its axis of revolution is obtained with very good accuracy for all values of the slip parameter $\beta b / \eta$ within the aspect-ratio range $0.5 \leqslant a / b \leqslant 2$. In addition, this method of semi-separation of variables incorporated with a boundary-collocation technique can be used to obtain numerical solutions for the same motion convergent and correct for arbitrary values of $\beta b / \eta$ and $a / b$. These collocation solutions are much superior to those resulting from a method of distributed internal singularities (Keh and Huang, 2004), in which the convergence behavior is relatively poor and no solution could be obtained outside the aspect-ratio range $0.2 \leqslant a / b \leqslant 5$. Our approach and results can be very useful for the calculations of the thermophoretic or photophoretic mobility of an aerosol spheroid (Talbot et al., 1980; Leong, 1984; Keh and Tu, 2001; Keh and Ou, 2004; Ou and Keh, 2005) in which both frictional and thermal slip velocities occur at the particle surface.

\section{Acknowledgement}

Part of this research was supported by the National Science Council of the Republic of China.

\section{Appendix A. Analytical solution for the coefficients in Eq. (12) truncated after two terms}

When Eq. (12) for the Stokes stream function is truncated after two terms $(N=2)$,

$$
\Psi=g_{2}(\lambda) G_{2}(\omega)+g_{4}(\lambda) G_{4}(\omega),
$$

where $g_{2}(\lambda)$ and $g_{4}(\lambda)$ are expressed by Eqs. (13) with $C_{2}=-2$ and $A_{4}=C_{4}=A_{6}=0$, the boundary conditions (15) and (23) for $n=2$ and 4 become

$$
\begin{gathered}
g_{2}\left(\lambda_{0}\right)=A_{1} G_{1}\left(\lambda_{0}\right)-2 G_{2}\left(\lambda_{0}\right)+D_{2} H_{2}\left(\lambda_{0}\right) \\
\quad+B_{4} H_{4}\left(\lambda_{0}\right)=0, \\
g_{4}\left(\lambda_{0}\right)=B_{4} H_{2}\left(\lambda_{0}\right)+D_{4} H_{4}\left(\lambda_{0}\right)+B_{6} H_{6}\left(\lambda_{0}\right)=0, \\
\frac{\eta}{\beta c}\left\{\left(\lambda_{0}^{2}-\gamma_{2}\right)\left[2+D_{2} H_{2}^{\prime \prime}\left(\lambda_{0}\right)+B_{4} H_{4}^{\prime \prime}\left(\lambda_{0}\right)\right]\right. \\
+2 \lambda_{0}\left[A_{1}-2 \lambda_{0}-D_{2} H_{2}^{\prime}\left(\lambda_{0}\right)-B_{4} H_{4}^{\prime}\left(\lambda_{0}\right)\right] \\
\left.\quad-\alpha_{4}\left[B_{4} H_{2}^{\prime \prime}\left(\lambda_{0}\right)+D_{4} H_{4}^{\prime \prime}\left(\lambda_{0}\right)+B_{6} H_{6}^{\prime \prime}\left(\lambda_{0}\right)\right]\right\} \\
=s_{22}\left[-A_{1}+2 \lambda_{0}+D_{2} H_{2}^{\prime}\left(\lambda_{0}\right)+B_{4} H_{4}^{\prime}\left(\lambda_{0}\right)\right] \\
+s_{42}\left[B_{4} H_{2}^{\prime}\left(\lambda_{0}\right)+D_{4} H_{4}^{\prime}\left(\lambda_{0}\right)+B_{6} H_{6}^{\prime}\left(\lambda_{0}\right)\right], \\
\frac{\eta}{\beta c}\left\{\left(\lambda_{0}^{2}-\gamma_{4}\right)\left[B_{4} H_{2}^{\prime \prime}\left(\lambda_{0}\right)+D_{4} H_{4}^{\prime \prime}\left(\lambda_{0}\right)+B_{6} H_{6}^{\prime \prime}\left(\lambda_{0}\right)\right]\right. \\
\quad-2 \lambda_{0}\left[B_{4} H_{2}^{\prime}\left(\lambda_{0}\right)+D_{4} H_{4}^{\prime}\left(\lambda_{0}\right)+B_{6} H_{6}^{\prime}\left(\lambda_{0}\right)\right] \\
\left.-\beta_{2}\left[2+D_{2} H_{2}^{\prime \prime}\left(\lambda_{0}\right)+B_{4} H_{4}^{\prime \prime}\left(\lambda_{0}\right)\right]\right\} \\
=s_{24}\left[-A_{1}+2 \lambda_{0}+D_{2} H_{2}^{\prime}\left(\lambda_{0}\right)+B_{4} H_{4}^{\prime}\left(\lambda_{0}\right)\right] \\
+s_{44}\left[B_{4} H_{2}^{\prime}\left(\lambda_{0}\right)+D_{4} H_{4}^{\prime}\left(\lambda_{0}\right)+B_{6} H_{6}^{\prime}\left(\lambda_{0}\right)\right] .
\end{gathered}
$$

Here, a prime on $H_{n}(\lambda)$ denotes a differentiation with respect to $\lambda$, and

$$
\begin{aligned}
s_{22}= & \frac{1}{\sqrt{\lambda_{0}^{2}-1}}\left(\frac{1}{8} \mathrm{e}^{3 \xi_{0}}+\frac{9}{40} \mathrm{e}^{\xi_{0}}+\frac{81}{280} \mathrm{e}^{-\xi_{0}}\right. \\
& \left.+\frac{71}{840} \mathrm{e}^{-3 \xi_{0}}+\frac{6}{385} \mathrm{e}^{-5 \xi_{0}}-\frac{18}{5005} \mathrm{e}^{-7 \xi_{0}}+\cdots\right), \\
s_{24}= & \frac{1}{\sqrt{\lambda_{0}^{2}-1}}\left(-\frac{3}{5} \mathrm{e}^{\xi_{0}}-\frac{1}{5} \mathrm{e}^{-\xi_{0}}-\frac{12}{55} \mathrm{e}^{-3 \xi_{0}}\right. \\
& \left.+\frac{36}{715} \mathrm{e}^{-5 \xi_{0}}+\frac{4}{715} \mathrm{e}^{-7 \xi_{0}}+\cdots\right), \\
s_{42}= & \frac{1}{\sqrt{\lambda_{0}^{2}-1}}\left(-\frac{3}{70} \mathrm{e}^{\xi_{0}}-\frac{1}{70} \mathrm{e}^{-\xi_{0}}-\frac{6}{385} \mathrm{e}^{-3 \xi_{0}}\right. \\
& \left.+\frac{18}{5005} \mathrm{e}^{-5 \xi_{0}}+\frac{2}{5005} \mathrm{e}^{-7 \xi_{0}}+\cdots\right), \\
s_{44}= & \frac{1}{\sqrt{\lambda_{0}^{2}-1}}\left(\frac{1}{8} \mathrm{e}^{3 \xi_{0}}+\frac{1}{40} \mathrm{e}^{\xi_{0}}+\frac{117}{440} \mathrm{e}^{-\xi_{0}}\right. \\
& \left.+\frac{31}{17160} \mathrm{e}^{-3 \xi_{0}}+\frac{16}{715} \mathrm{e}^{-5 \xi_{0}}+\frac{48}{12155} \mathrm{e}^{-7 \xi_{0}}+\cdots\right) .
\end{aligned}
$$

Note that all $s_{m n}$ are convergent for a given value of $\xi_{0}$.

Eqs. (A.2)-(A.5) with the terms involving the coefficient $B_{6}$ being neglected are used to determine the coefficients $A_{1}$, $D_{2}, B_{4}$, and $D_{4}$, and their result in explicit forms is

$$
\begin{aligned}
A_{1}= & \left\{\left(f_{c} k_{23}-f_{2} k_{c 3}\right) H_{4}\left(\lambda_{0}\right)+k_{4} H_{2}\left(\lambda_{0}\right)\left[f_{c} H_{2}\left(\lambda_{0}\right)\right.\right. \\
& \left.\left.+f_{2}\left(\lambda_{0}^{2}-1\right)\right]+f_{34} k_{c 2}\right\} \Gamma, \\
D_{2}= & \left\{\left(f_{1} k_{c 3}-f_{c} k_{13}\right) H_{4}\left(\lambda_{0}\right)+k_{4} H_{2}\left(\lambda_{0}\right)\left[f_{c} \lambda_{0}\right.\right. \\
& \left.\left.-f_{1}\left(\lambda_{0}^{2}-1\right)\right]+f_{34} k_{c 1}\right\} \Gamma,
\end{aligned}
$$




$$
\begin{aligned}
& B_{4}=-H_{4}\left(\lambda_{0}\right)\left(f_{c} k_{12}-f_{2} k_{c 1}-f_{1} k_{c 2}\right) \Gamma, \\
& D_{4}=-\frac{H_{2}\left(\lambda_{0}\right)}{H_{4}\left(\lambda_{0}\right)} B_{4},
\end{aligned}
$$

where

$$
\begin{aligned}
\Gamma= & \left\{\left(f_{1} k_{23}-f_{2} k_{13}\right) H_{4}\left(\lambda_{0}\right)+k_{4} H_{2}\left(\lambda_{0}\right)\left[f_{1} H_{2}\left(\lambda_{0}\right)\right.\right. \\
& \left.\left.+f_{2} \lambda_{0}\right]+f_{34} k_{12}\right\}^{-1}, \\
f_{1}= & s_{22}+\frac{\eta}{\beta c} 2 \lambda_{0}, \\
f_{2}= & -s_{22} H_{2}^{\prime}\left(\lambda_{0}\right)+\frac{\eta}{\beta c}\left[\left(\lambda_{0}^{2}-\gamma_{2}\right) H_{2}^{\prime \prime}\left(\lambda_{0}\right)\right. \\
& \left.-2 \lambda_{0} H_{2}^{\prime}\left(\lambda_{0}\right)\right], \\
f_{3}= & -s_{42} H_{2}^{\prime}\left(\lambda_{0}\right)-s_{22} H_{4}^{\prime}\left(\lambda_{0}\right)+\frac{\eta}{\beta c}\left[\left(\lambda_{0}^{2}-\gamma_{2}\right) H_{4}^{\prime \prime}\left(\lambda_{0}\right)\right. \\
& \left.-2 \lambda_{0} H_{4}^{\prime}\left(\lambda_{0}\right)-\alpha_{4} H_{2}^{\prime \prime}\left(\lambda_{0}\right)\right], \\
f_{4}= & -s_{42} H_{4}^{\prime}\left(\lambda_{0}\right)-\frac{\eta}{\beta c} \alpha_{4} H_{4}^{\prime \prime}\left(\lambda_{0}\right), \\
f_{c}= & 2\left[s_{22} \lambda_{0}+\frac{\eta}{\beta c}\left(\lambda_{0}^{2}+\gamma_{2}\right)\right], \\
k_{1}= & s_{24}, \\
k_{2}= & -s_{24} H_{2}^{\prime}\left(\lambda_{0}\right)-\frac{\eta}{\beta c} \beta_{2} H_{2}^{\prime \prime}\left(\lambda_{0}\right), \\
k_{3}= & -s_{44} H_{2}^{\prime}\left(\lambda_{0}\right)-s_{24} H_{4}^{\prime}\left(\lambda_{0}\right)+\frac{\eta}{\beta c}\left[\left(\lambda_{0}^{2}-\gamma_{4}\right) H_{2}^{\prime \prime}\left(\lambda_{0}\right)\right. \\
& \left.-2 \lambda_{0} H_{2}^{\prime}\left(\lambda_{0}\right)-\beta_{2} H_{4}^{\prime \prime}\left(\lambda_{0}\right)\right], \\
k_{4}= & -s_{44} H_{4}^{\prime}\left(\lambda_{0}\right)+\frac{\eta}{\beta c}\left[\left(\lambda_{0}^{2}-\gamma_{4}\right) H_{4}^{\prime \prime}\left(\lambda_{0}\right)\right. \\
& \left.-2 \lambda_{0} H_{4}^{\prime}\left(\lambda_{0}\right)\right], \\
k_{c}= & 2\left(s_{24} \lambda_{0}+\frac{\eta}{\beta c} \beta_{2}\right), \\
f_{34}= & f_{3} H_{4}\left(\lambda_{0}\right)-f_{4} H_{2}\left(\lambda_{0}\right), \\
k_{12}= & k_{1} H_{2}\left(\lambda_{0}\right)+k_{2} \lambda_{0}, \\
k_{13}= & k_{1} H_{4}\left(\lambda_{0}\right)+k_{3} \lambda_{0}, \\
k_{23}= & k_{2} H_{4}\left(\lambda_{0}\right)-k_{3} H_{2}\left(\lambda_{0}\right), \\
k_{c 1}= & k_{c} \lambda_{0}-k_{1}\left(\lambda_{0}^{2}-1\right), \\
k_{c 2}= & k_{c} H_{2}\left(\lambda_{0}\right)+k_{2}\left(\lambda_{0}^{2}-1\right), \\
k_{c 3}= & k_{c} H_{4}\left(\lambda_{0}\right)+k_{3}\left(\lambda_{0}^{2}-1\right) . \\
& \\
&
\end{aligned}
$$

An explicit expression for the hydrodynamic drag force acting on a spheroid translating along its axis of revolution results from Eqs. (19) and (A.7a). After the substitution of Eq. (A.7a) into Eq. (19) taking $\beta b / \eta \rightarrow \infty$ and $\beta b / \eta \rightarrow 0$, we obtain, respectively, the explicit formula for the drag force acting on a no-slip spheroid given in Happel and Brenner (1983) and the following expression for the hydrodynamic force exerted on a perfectly slip spheroid:

$F=-4 \pi \eta b U \frac{X\left(\lambda_{0}\right)}{Y\left(\lambda_{0}\right)}$

where

$$
\begin{aligned}
X\left(\lambda_{0}\right)= & 28 \sqrt{\lambda_{0}^{2}-1}\left[\lambda_{0}^{2}\left(36 \lambda_{0}^{4}-63 \lambda_{0}^{2}+29\right)\right. \\
& -2 \lambda_{0}\left(36 \lambda_{0}^{6}-75 \lambda_{0}^{4}+47 \lambda_{0}^{2}-8\right) \operatorname{coth}^{-1} \lambda_{0} \\
& \left.+3\left(\lambda_{0}^{2}-1\right)^{2}\left(12 \lambda_{0}^{4}-5 \lambda_{0}^{2}+1\right)\left(\operatorname{coth}^{-1} \lambda_{0}\right)^{2}\right],
\end{aligned}
$$

$$
\begin{aligned}
Y\left(\lambda_{0}\right)= & -\lambda_{0}^{3}\left(189 \lambda_{0}^{6}-819 \lambda_{0}^{4}+1035 \lambda_{0}^{2}-421\right) \\
& +\lambda_{0}^{2}\left(567 \lambda_{0}^{8}-1638 \lambda_{0}^{6}+1320 \lambda_{0}^{4}-10 \lambda_{0}^{2}-239\right) \operatorname{coth}^{-1} \lambda_{0} \\
& -3 \lambda_{0}\left(\lambda_{0}^{2}-1\right)^{2}\left(189 \lambda_{0}^{6}+105 \lambda_{0}^{4}-337 \lambda_{0}^{2}+67\right)\left(\operatorname{coth}^{-1} \lambda_{0}\right)^{2} \\
& +9\left(\lambda_{0}^{2}-1\right)^{3}\left(21 \lambda_{0}^{6}+63 \lambda_{0}^{4}-25 \lambda_{0}^{2}+5\right)\left(\operatorname{coth}^{-1} \lambda_{0}\right)^{3} .
\end{aligned}
$$

Note that, in the limiting cases of $\beta b / \eta \rightarrow \infty$ and $\beta b / \eta \rightarrow 0$, all $s_{m n}$ in Eq. (A.6) will cancel out in the solution of the coefficients $A_{1}, D_{2}, B_{4}$, and $D_{4}$. In the limit $a / b \rightarrow \infty$, Eq. (A.10) (and the corresponding expressions for cases of $N>2$ ) leads to $F=0$.

\section{References}

Basset, A.B., 1961. In: A Treatise on Hydrodynamics, vol. 2. Dover, New York.

Batchelor, G.K., 1970. Slender-body theory for particles of arbitrary cross-section in Stokes flow. J. Fluid Mech. 44, 419-440.

Beavers, G.S., Joseph, D.D., 1967. Boundary conditions at a naturally permeable wall. J. Fluid Mech. 30, 197-207.

Brenner, H., 1964. The Stokes resistance of a slightly deformed sphere. Chem. Eng. Sci. 19, 519-539.

Cox, R.G., 1970. The motion of long slender bodies in a viscous fluid, Part 1. General theory. J. Fluid Mech. 44, 791-810.

Dahneke, B.E., 1973. Slip correction factors for nonspherical bodies-III. The form of the general law. J. Aerosol Sci. 4, 163-170.

Dassios, G., Hadjinicolaou, M., Payatakes, A.C., 1994. Generalized eigenfunctions and complete semiseparable solutions for Stokes flow in spheroidal coordinates. Quart. Appl. Math. 52, 157-191.

Dassios, G., Hadjinicolaou, M., Coutelieris, F.A., Payatakes, A.C., 1995. Stokes flow in spheroidal particle-in-cell models with Happel and Kuwabara boundary conditions. Int. J. Eng. Sci. 33, 1465-1490.

Davis, M.H., 1972. Collisions of small cloud droplets: gas kinetic effects. J. Atmos. Sci. 29, 911-915.

Deo, S., Datta, S., 2002. Slip flow past a prolate spheroid. Indian J. Pure Appl. Math. 33, 903-909.

Gluckman, M.J., Pfeffer, R., Weinbaum, S., 1971. A new technique for treating multi-particle slow viscous flow: axisymmetric flow past spheres and spheroids. J. Fluid Mech. 50, 705-740.

Gogte, S., Vorobieff, P., Truesdell, R., Mammoli, A., van Swol, F., Shah, P., Brinker, C.J., 2005. Effective slip on textured superhydrophobic surfaces. Phys. Fluids 17, 051701.

Goren, S.L., O’Neill, M.E., 1980. Asymmetric creeping motion of an open torus. J. Fluid Mech. 101, 97-110.

Happel, J., Brenner, H., 1983. Low Reynolds Number Hydrodynamics. Nijhoff, Dordrecht, The Netherlands.

Hutchins, D.K., Harper, M.H., Felder, R.L., 1995. Slip correction measurements for solid spherical particles by modulated dynamic light scattering. Aerosol Sci. Technol. 22, 202-218.

Jones, I.P., 1973. Low Reynolds number flow past a porous spherical shell. Proc. Cambridge Philos. Soc. 73, 231-238.

Keh, H.J., Chen, S.H., 1996. The motion of a slip spherical particle in an arbitrary Stokes flow. Eur. J. Mech., B/Fluids 15, 791-807.

Keh, H.J., Huang, C.H., 2004. Slow motion of axisymmetric slip particles along their axes of revolution. Int. J. Eng. Sci. 42, 1621-1644. 
Keh, H.J., Ou, C.L., 2004. Thermophoresis of aerosol spheroids. Aerosol Sci. Technol. 38, 675-684.

Keh, H.J., Shiau, S.C., 2000. Effects of inertia on the slow motion of aerosol particles. Chem. Eng. Sci. 42, 1621-1644.

Keh, H.J., Tu, H.J., 2001. Thermophoresis and photophoresis of cylindrical particles. Colloids Surf., A 176, 213-223.

Kennard, E.H., 1938. Kinetic Theory of Gases. McGraw-Hill, New York.

Leong, K.H., 1984. Thermophoresis and diffusiophoresis of large aerosol particles of different shapes. J. Aerosol Sci. 15, 511-517.

Nir, A., 1976. Linear shear flow past a porous particle. Appl. Sci. Res. 32, 313-325.

Oberbeck, A., 1876. Uber stationare Flussigkeitsbewegungen mit Berucksichtigung der inner Reibung. J. Reine Angew. Math. 81, 62-80.

O'Brien, V., 1968. Form factors for deformed spheroids in Stokes flow. A.I.Ch.E.J. 14, 870-875.

Ou, C.L., Keh, H.J., 2005. Low Knudsen number photophoresis of aerosol spheroids. J. Colloid Interface Sci. 282, 69-79.

Palaniappan, D., 1994. Creeping flow about a slightly deformed sphere. ZAMP 45, 832-838.
Payne, L.E., Pell, W.H., 1960. The Stokes flow problem for a class of axially symmetric bodies. J. Fluid Mech. 7, 529-549.

Ramkissoon, H., 1997. Slip flow past an approximate spheroid. Acta Mech. 123, 227-233.

Saffman, P.G., 1971. On the boundary condition at the surface of a porous medium. Studies Appl. Math. 50, 93-101.

Senchenko, S., Keh, H.J., 2006. Slipping Stokes flow around a slightly deformed sphere. Phys. Fluids 18, 088104.

Sharipov, F., Kalempa, D., 2003. Velocity slip and temperature jump coefficients for gaseous mixtures. I. Viscous slip coefficient. Phys. Fluids 15, 1800-1806.

Stokes, G.G., 1851. On the effect of the internal friction of fluid on pendulums. Trans. Cambridge Philos. Soc. 9, 8-106.

Talbot, L., Cheng, R.K., Schefer, R.W., Willis, D.R., 1980. Thermophoresis of particles in heated boundary layer. J. Fluid Mech. 101, 737-758.

Tretheway, D.C., Meinhart, C.D., 2002. Apparent fluid slip at hydrophobic microchannel walls. Phys. Fluids 14, L9-L12.

Williams, M.M.R., 1986. Thermophoretic forces acting on a spheroid. J. Phys. D: Appl. Phys. 19, 1631-1642. 